\title{
Der niederste optische Anregungszustand von Hexamethylbenzol-Kristallen
}

\author{
Von Hans Christoph Wolf \\ Aus dem II. Physikalischen Institut der Techn. Hochschule Stuttgart \\ (Z. Naturforschg. 13 a, 336 -345 [1958] ; eingegangen am 20. Januar 1958)
}

\begin{abstract}
Das Absorptions- und Fluoreszenzspektrum von Hexamethylbenzol-Einkristallen wurde bei $77^{\circ} \mathrm{K}$ senkrecht zur $b c$-Ebene mit polarisiertem Licht photographisch aufgenommen. Dabei wurden in Übereinstimmung mit BRoude 2 Modifikationen beobachtet; diejenige mit bekannter Kristallstruktur ist unterhalb ca. $110^{\circ} \mathrm{K}$ instabil, kann aber auch dort bei raschem Unterkühlen in sehr dünnen Kristallen beobachtet werden. Die linienreichen Spektren bestehen in der Hauptsache aus 2 etwa gleich intensiven Serien. Eine M-Serie entspricht dem Spektrum des freien Moleküls; sie ist Sym. metrie-verboten und erhält ihre Intensität durch eine $E_{\mathrm{g}}$-Schwingung. Das Übergangsmoment für die Banden dieser Serie liegt in beiden Phasen in der Molekülebene.

Die andere Serie ist durch das Kristallfeld induziert (K-Serie) und hat die Struktur eines erlaubten Überganges. Das Übergangsmoment für die Banden dieser Serie liegt bei der Hochtemperaturphase weitgehend in der Molekülebene, bei der Tieftemperaturphase senkrecht zur Molekülebene. Es ist möglich, daß die Phasenänderung nur in einem Umklappen der $\mathrm{CH}_{3}$-Gruppen in eine andere Lage relativ zum Benzolring besteht.
\end{abstract}

Im Rahmen einer Untersuchung der UV-Spektren von Molekülkristallen wird im folgenden über spektroskopische Untersuchungen an HexamethylbenzolKristallen berichtet.

\section{Wahl des Stoffes}

Hexamethylbenzol (HMB) ist ein einfacher aromatischer Kohlenwasserstoff. Es kristallisiert mit nur einem Molekül in der Einheitszelle. Zudem ist mit ihm des höheren Schmelzpunktes wegen in vielen Fällen einfacher zu hantieren als mit Benzol selbst. Deshalb wurde HMB schon oft als Modellsubstanz zur Untersuchung physikalischer Eigenschaften des Benzolkernes und seiner $\pi$-Elektronen verwendet. So untersuchte LonSDALE ${ }^{1}$ die Kristallstruktur besonders zur Klärung der Frage, ob der Ring eben ist und ob die Bindungsverhältnisse mehr an Diamant oder Graphit erinnern. Daran anschließend untersuchte Bhagavantam ${ }^{2}$ an HMB die optische und magnetische Anisotropie des Benzol-Ringes. Von Scheibe und Mitarbeitern ${ }^{3}$ wurde an HMB-Kristallen gezeigt, daß die optische Anregung der $\pi$-Elektronen vorzugsweise in der Ring-Ebene erfolgt. Schließlich diente eine genaue Analyse der Kristallstruktur von Brockway und Robertson ${ }^{4}$ der Messung der $\mathrm{CH}_{3}$ Bindungslänge an Benzolkernen.

1 K. Lonsdale, Proc. Roy. Soc., Lond. A 123, 494 [1929].

2 S. Bhagavantam, Proc. Roy. Soc., Lond. A 126, 143 [1930].

3 G. Scheibe, St. Hartwig u. R. Müller, Z. Elektrochem. 49, 376 [1943].

\section{Frühere spektroskopische Arbeiten}

Die erste UV-spektroskopische Untersuchung von HMB-Kristallen ist die erwähnte Messung der HMBKristall-Absorption im UV von Scheibe, Hartwig und MüLleR ${ }^{3}$. Da bei Raumtemperatur gemessen wurde, erlaubt diese Messung im wesentlichen nur Aussagen über die integrale Absorption in zwei Kristallrichtungen, keine Analyse der Schwingungsstruktur. Das gleiche gilt für die Arbeit von NeLson ${ }^{5}$. Um weiter zu kommen, benötigt man Messungen mit hoher Auflösung in polarisiertem Licht bei tiefen Temperaturen. Diesen Schritt tat zuerst Broude ${ }^{6}$. Das vollständige Absorptions- und Fluoreszenzspektrum des HMB-Kristalls bei $20^{\circ} \mathrm{K}$ photographierte und analysierte McClure ${ }^{7}$. Jedoch handelt es sich bei den von McClure gemessenen Spektren, wie unten gezeigt wird, um die Spektren der Tieftemperatur-Modifikation des HMB, deren Kristallstruktur unbekannt ist. Die im folgenden mitgeteilten Messungen an HMB-Kristallen bestätigen für diese Modifikation lediglich die Messungen von McClure. Darüber hinaus wurde jedoch auch das Spektrum der bei $77^{\circ} \mathrm{K}$ instabilen Hochtemperatur-Modifikation mit bekannter Kristallstruktur aufgenommen.

4 L. O. Brockway u. J. M. Robertson, J. Chem. Soc. 1939, 1324.

5 R.C.Nelson u. W.T.Simpson, J.Chem. Phys. 23, 1146[1955].

${ }_{6}$ W. L. Broude, Zhurn. eksp. teoret. Fiz. 22, 600 [1952].

7 O.Schnepp u. D.S. McClure, J. Chem. Phys. 26, 83 [1957]. 


\section{Die Kristallstruktur}

Wesentlicher Grund für die Untersuchung von HMB ist, wie erwähnt, seine einfache Kristallstruktur: nur ein Molekül in der Einheitszelle. Der Kristall ist triklin mit folgenden Daten:

$$
\begin{aligned}
a & =8,92 \pm 0,02 \AA \\
b & =8,86 \pm 0,02 \AA \\
c & =5,30 \pm 0,01 \AA \\
d_{100} & =7,66 \AA \\
d_{010} & =5,96 \AA \\
d_{001} & =3,66 \AA
\end{aligned}
$$

Tab. 1. Kristall-Daten von HMB.

Man erhält bei der Züchtung dünner Kristallplatten nach den üblichen Methoden meist Kristalle mit der Fläche $b c$ als Oberfläche. Nach Tab. 1 steht die Ebene der Moleküle auf dieser Fläche fast, jedoch nicht genau senkrecht; der Winkel beträgt ca. $80^{\circ}$ (siehe Abb. 1). Dabei liegt die Molekülebene ungefähr in der $b$-Achse. Diese Achse ist zudem optische Achse.

Von Broude und McClure wurden auch dünne Kristalle mit der Fläche $a b$ untersucht; mit dieser Fläche fällt die Molekülebene annähernd zusammen. Die $b$-Achse des Kristalls fällt annähernd mit einer der dielektrischen Hauptachsen zusammen, die $c^{\prime}$. Achse mit einer anderen. Da im Sichtbaren eine Dispersion der Achsen fehlt, kann angenommen werden, daß dies auch im Ultravioletten gilt.

\section{Kristallzucht und Orientierung}

Aus Benzol-Lösung erhält man große, tafelförmige Einkristalle mit wohl ausgebildeten Flächen, besonders der Fläche $b c$. Ihre Dicke beträgt ca. 25 bis $200 \mu$. Dünnere Kristalle wurden zunächst durch Erstarrenlassen der HMB-Schmelze zwischen Platten aus Kristallquarz oder Quarzglas unter Druck hergestellt. Die so erreichbare Dicke liegt je nach Druck

Abb. 1. Lage des HMB-Moleküls in der Einheitszelle; links $=b c$-Ebene, rechts $=a b$-Ebene.

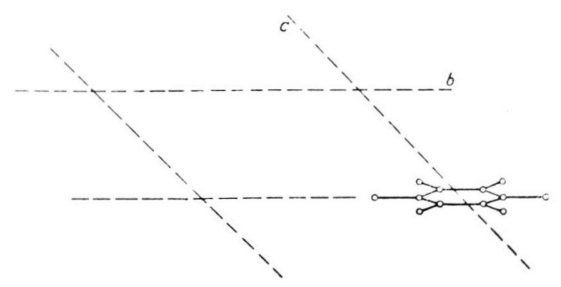

(ca. $50 \mathrm{~g}$ bis $50 \mathrm{~kg} / \mathrm{cm}^{2}$ ) zwischen ca. 25 und $2 \mu$. Diese Preßplatten waren jedoch in ihrer Orientierung wenig einheitlich; sie bestanden aus einem Konglomerat kleiner, verschieden orientierter Kristallbereiche. Wie Broude gezeigt hat, kann man daraus durch Tempern größere Einkristalle erhalten. Dieses Verfahren wurde hier nicht angewandt, es wurde vielmehr auf das bei Naphthalin bewährte Verfahren ${ }^{9}$ zurückgegriffen: Eine Lösung von HMB in Benzol ergibt bei langsamem Verdunsten auf einer Wasseroberfläche je nach der Konzentration Kristalle von ca. 0,5 bis ca. $30 \mu$ Dicke in Flächen von $\mathrm{cm}^{2}$. Die dünnen HMB-Kristalle sind außerordentlich leicht elastisch und plastisch verformbar.

Die Orientierung der Kristalle ist einfach: Die äußere Begrenzung der dünnen Kristalle mit $b c$ Flächen sind im allgemeinen die Kanten $b$ und $c$; man stellt dies leicht durch Messung der Parallelogramm-Winkel an den Kristallen fest. Ferner ist außer der $b c$-Ebene auch die Ebene $a b$ Spaltebene. Dies macht sich in der $b c$-Ebene durch feine Spaltrisse parallel der Kante $b$ bemerkbar, besonders ausgeprägt beim Abkühlen der Kristalle auf Tieftemperatur. Schließlich erhält man zwischen gekreuzten Nicols als Auslöschrichtungen die Richtungen $b$ und $c^{\prime}$ (senkrecht zu $b$ in der $b c$-Ebene). Das konoskopische Bild der $b c$-Ebene ist dagegen wenig charakteristisch und wurde nicht zur Orientierung benutzt.

Die Dicke der Kristalle wurde unterhalb ca. $10 \mu$ aus den Interferenzfarben zwischen gekreuzten Nicols, oberhalb $10 \mu$ aus ihrem Gewicht bestimmt.

\section{Die Versuchsanordnung}

Die Kristalle wurden auf dünnen Platten aus Quarzglas aufgebracht. Diese wurden in einem Küvettenhalter befestigt, der zur Kühlung mit flüssigem Sauerstoff oder Stickstoff gefüllt und zur Wärme-Isolierung in ein Quarz-Dewar-Gefäß eingesetzt werden konnte. Die Ver-

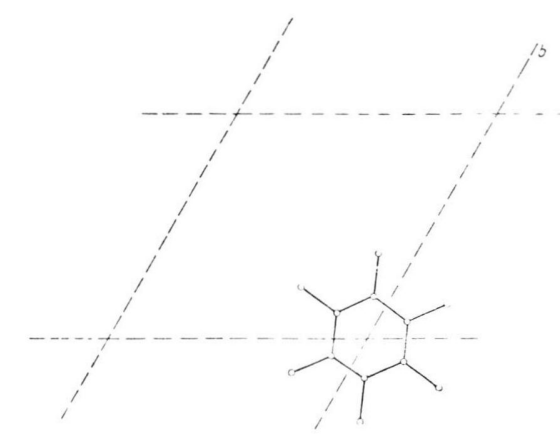


suchsanordnung zeigt Abb. 2. Das Kontinuum einer $\mathrm{X}$ - oder $\mathrm{H}_{2}$-Lampe fällt parallel durch den Kristall und ein Glan-Prisma mit Luftspalt; die Linse $\mathrm{L}_{2}$ bildet den Kristall unscharf und etwa 3 -fach vergrößert auf den Spalt des Spektrographen ab. Die unscharfe Abbildung wurde gewählt, um Streifen in den Spektren wegen kleiner Kristallfehler zu unterdrücken. Das Glan-Prisma ist senkrecht zum Strahlengang um kleine Beträge verschiebbar. Dies ist zur Korrektur einer geringen Strahlversetzung bei der Drehung des Glan-Prismas nötig.

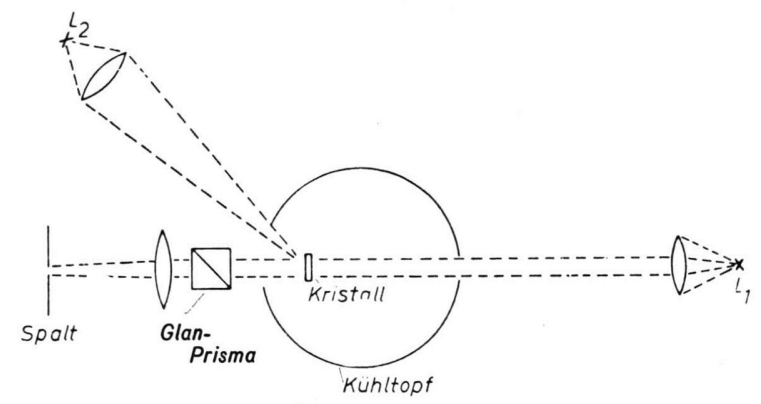

Abb. 2. Schema der Meßanordnung.

Die Spektren wurden photographisch aufgenommen. Die Photoplatten konnten mit einem Registrierphotometer ausgewertet werden *. Als Referenzlinie dienten $\mathrm{Hg}$-Linien, besonders die auch im X-Kontinuum schwach sichtbare Hg-Linie 2536,5 A. Die Genauigkeit in der Messung der Wellenlängen lag bei $\pm 5 \mathrm{~cm}^{-1}$.

Die Messungen wurden im allgemeinen bei der Temperatur des flüssigen $\mathrm{N}_{2}$ durchgeführt $\left(77^{\circ} \mathrm{K}\right)$. Bei dieser Temperatur sind die Spektren deutlich schärfer als bei der des flüssigen Sauerstoffs.

Zur Beobachtung der Fluoreszenz wurde der Kristall schräg von vorne mit dem Kontinuum der X-Lampe bestrahlt, aus dem durch das auch früher schon benutzte Cyanin-Filter (8) der Wellenlängenbereich von ca. 275 bis ca. $350 \mathrm{~m} \mu$ herausgefiltert war. Das Fluoreszenzlicht wurde durch eine Linse kurzer Brennweite zum Durchgang durch das Glan-Prisma parallel gemacht und von einer zweiten Linse wieder unscharf auf den Spektrographen-Spalt abgebildet.

Die Phosphoreszenz wurde nur visuell beobachtet.

\section{Reinigung}

Das verwendete HMB stammte aus dem Physikal.Chem. Institut der T.H. München (von Herrn Dr. DörR), aus dem Organ.-Chem. Institut der T.H. Stuttgart (von Herrn Dr. Gompper) und aus dem Ammoniaklaboratorium der BASF Ludwigshafen (von Herrn Dr. Schlenk). Nach nochmaliger chromatographischer Reinigung stimmten die 3 Proben in ihren Absorptions-, Fluoreszenzund Phosphoreszenz-Spektren völlig überein. Die Substanzen waren als spektroskopisch rein zu bezeichnen.

* Herrn Prof. G. Scheibe bin ich für die Erlaubnis zur Benutzung des Registrierphotometers im Physikalisch-Chemischen Institut der TH München zu großem Dank verpflichtet.

\section{Experimentelle Ergebnisse}

a) Die Lösungs-Spektren

Zum Verständnis des Kristallspektrums hatte es sich bei Naphthalin ${ }^{8,9}$ bewährt, vom Lösungsspektrum auszugehen, um den Beginn des Spektrums (die 0.0-Bande) und die Schwingungsstruktur im groben festzulegen. Abb. 3 zeigt das Absorptions-

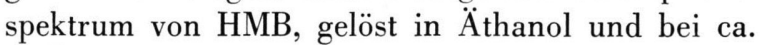
$95{ }^{\circ} \mathrm{K}$ glasartig erstarrt. Die Banden sind auch bei Tieftemperatur noch relativ breit; die Lage der breiten Maxima gibt Tab. 2.

\begin{tabular}{|c|c|c|}
\hline Nr. & $\begin{array}{c}\text { Wellenzahl } \\
\mathrm{cm}^{-1}\end{array}$ & Zuordnung \\
\hline 1 & $?(35400)$ & 0,0 \\
2 & 35820 & $0,0+420$ \\
3 & 36400 & $2+580$ \\
4 & 36950 & $2+1130$ \\
5 & 37550 & $4+600$ \\
6 & 38200 & $4+1250$ \\
7 & ca. 38700 & $6+500$ \\
8 & 39400 & $6+1200$ \\
9 & 40000 & $8+600$ \\
10 & 40600 & $8+1200$ \\
11 & 41200 & $10+600$ \\
\hline
\end{tabular}

Tab. 2. Absorptionsspektrum von HMB in Äthanol bei $95^{\circ} \mathrm{K}$. Diesem Spektrum ist bei Zimmertemperatur noch eine Absorptionsbande ( $1-0$-Bande) vorgelagert. Meßgenauigkeit ca. $50 \mathrm{~cm}^{-1}$.

\begin{tabular}{|c|r|l|}
\hline Nr. & $\begin{array}{c}\text { Wellenzahl } \\
\mathrm{cm}^{-1}\end{array}$ & Zuordnung \\
\hline 1 & ca. 35400 & 0,0 \\
2 & 34875 & $0,0-525$ \\
3 & 34290 & $2-585$ \\
4 & 33660 & $2-1215$ \\
5 & 33100 & $4-560$ \\
6 & ca. 32455 & $4-1210$ \\
\hline
\end{tabular}

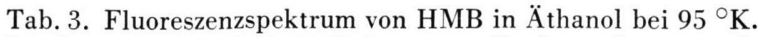
Die Lage der Bande 1 (0.0) ist wegen Reabsorption nur mit einem Fehler von \pm ca. $60 \mathrm{~cm}^{-1}$ anzugeben.

8 H. C. Wolf, Z. Naturforschg. 10 a, 3 [1955].

9 D.Griessbach, G. Will u. H.C. Wolf, Z. Naturforschg. 11 a, 791 [1956]. 


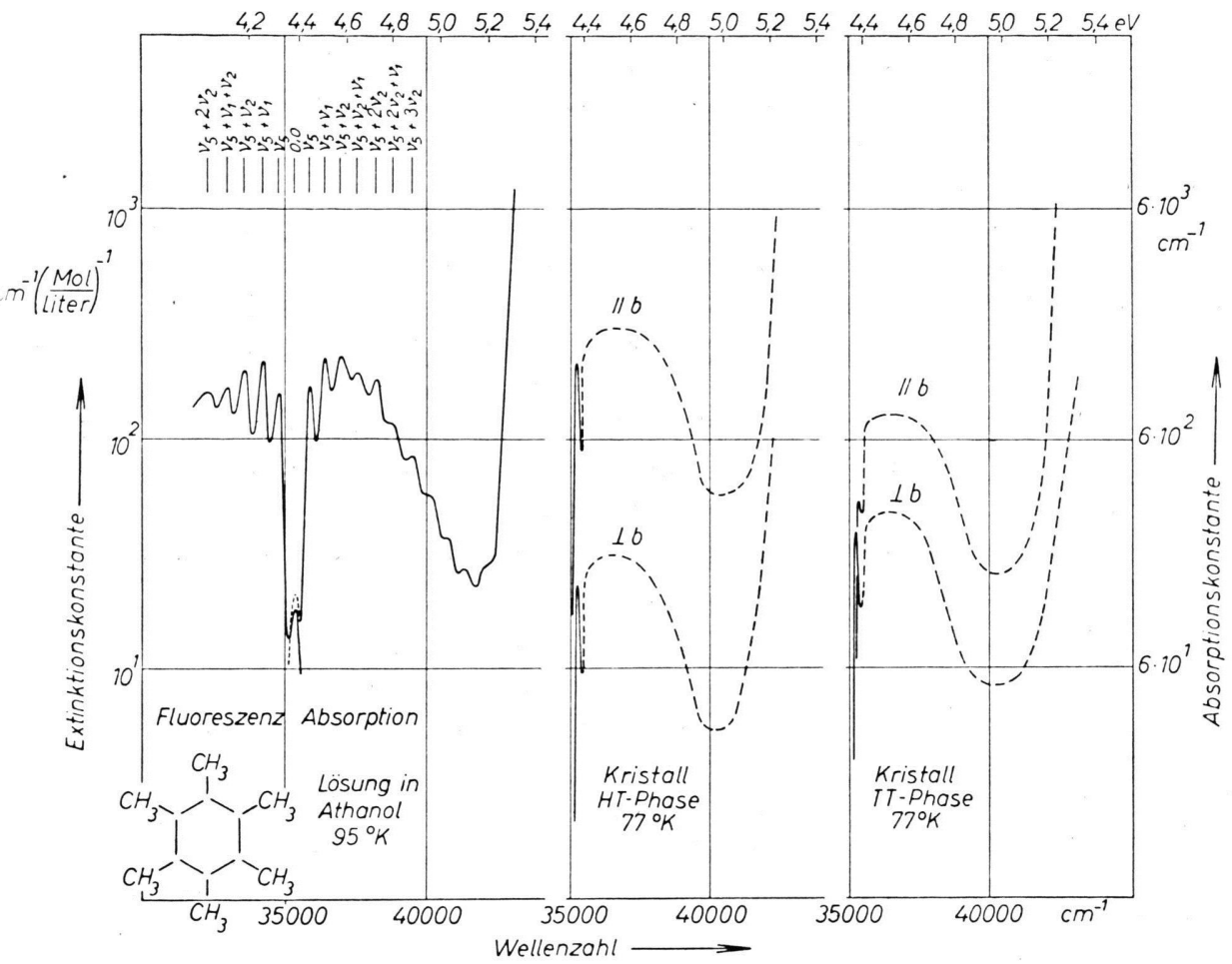

Abb. 3. links: Absorptions- und Fluoreszenzsnektrum von $\mathrm{HMB}$ in Äthanol (glasartig er. starrt) bei $95^{\circ} \mathrm{K}$; Mitte: Absorptionsspektrum der HT-Phase; 0.0-Linie und Umhüllende der übrigen Linien; rechts: Absorptionsspektrum der TT. Phase; 0.1-Linie parallel $b, 0.0$-Linie senkrecht $b$ und Umhüllende der übrigen Linien.

Tab. 3 gibt das Fluoreszenzspektrum. Die beiden Spektren werden dann in der von Molekülspektren gewohnten Weise verständlich, wenn man annimmt, daß die im Fluoreszenzspektrum als 0.0 erscheinende Bande ca. $35420 \mathrm{~cm}^{-1}$ auch dem Absorptionsspektrum vorzulagern ist. Durch die geringe Löslichkeit des $\mathrm{HMB}$ in Äthanol bei $100^{\circ} \mathrm{K}$ (Grenze bei ca. $10^{-3} \mathrm{~mol}$ ) und die durch die Apparatur gegebene Begrenzung in der größten Schichtdicke, bei der gemessen werden konnte, wird die Messung von Extinktionskoeffizienten, die kleiner als ca. $30 \mathrm{~cm}^{-1}$ $(\mathrm{Mol} / \text { Liter })^{-1}$ sind, unmöglich. Dies ist damit die obere Grenze für den $\varepsilon$-Wert der 0.0-Bande. Durch das Kristallspektrum findet die Annahme einer vorgelagerten 0.0-Bande später noch ihre endgültige Bestätigung.

Mit der Wahl dieser 0.0-Bande ergibt sich eine sinnvolle Zuordnung der beobachteten Schwingungsbanden. Die Zuordnung schließt sich wegen der großen Ähnlichkeit der Spektren und der Molekülsymmetrie an die bei Benzol bewährte an, ist aber wegen der großen Bandenbreite nicht so gut gesichert. 0.0 ist sehr schwach, der 1. Übergang Symmetrieverboten. Eine Molekülschwingung mit der Frequenz ca. $500 \mathrm{~cm}^{-1}$ im Grundzustand gibt dem Übergang seine Intensität. Vermutlich ist das die $E_{\mathrm{g}}$-Frequenz
$508 \mathrm{~cm}^{-1}$ des Raman-Spektrums (Tab. 6), im folgenden als $v_{5}$ bezeichnet. Dazu addieren sich zwei Schwingungsfrequenzen, die Perioden bilden: ca. 570 und ca. $1210 \mathrm{~cm}^{-1}$ im Grundzustand. Das sind vermutlich die $A_{\mathrm{g}}$-Frequenzen 561 und $1295 \mathrm{~cm}^{-1}$ des Raman-Spektrums; der Meßwert 1210 ist eine Überlagerung von $2 \times 561 \mathrm{mit} 1295 \mathrm{~cm}^{-1}$. Im folgenden heißen diese Schwingungen $v_{1}$ und $v_{2}$.

Die im Lösungsspektrum analysierbare „Molekülserie“ (M-Serie) des Hexamethylbenzol-Spektrums hat dann die folgende Formel:

$$
\begin{aligned}
\text { M-Serie }=0.0 \text { (verboten) } & \pm v_{5} \pm n_{1} v_{1} \pm n_{2} v_{2} \\
& \text { mit } n_{1,2}=0,1,2,3, \ldots .
\end{aligned}
$$

b) Die Kristall-Spektren

\section{Allgemeines}

Absorptionsspektren von HMB-Kristallen wurden gemessen: 1) an Einkristallen mit der Fläche $b c$, polarisiert parallel $b$ und $\left.c^{\prime} ; 2\right)$ an Preßplatten zwischen Quarzscheiben. Hier wurde wegen der Vielzahl verschieden orientierter Kristallbereiche unpolarisiert gemessen. Die Spektren der Preßplatten sind intensiver und homogener, weil Störungen durch Spaltrisse, nicht-ebene Oberflächen und andere Fehler der Einkristalle wegfallen. Deshalb können diese 
Spektren zur exakten Ausmessung von Linien dienen und haben damit ihren eigenen Wert auch neben denen der Einkristalle.

Die Linienbreite ist bei $77^{\circ} \mathrm{K}$ bereits so gering, daß man einen Teil der die Moleküllinien begleitenden Gitterschwingungs-Linien deutlich unterscheiden kann. Auf ihre Analyse (die sich zum Teil bei Mc Clure $^{7}$ findet) wird hier jedoch nicht eingegangen.

\section{Die Temperatur-Abhängigkeit}

Verfolgt man die Änderung des Kristall-Absorptionsspektrums während des Einfrierens, so beobachtet man eine kontinuierliche Verschärfung der bei Zimmertemperatur sehr breiten Absorptionsbanden. Die Lage der Bandenschwerpunkte bleibt dabei weitgehend erhalten (Abb. $4 \mathrm{a}^{*}$ ). Bemerkenswert ist, daß sich die Intensität der 0.0-Bande während des Einfrierens kontinuierlich verstärkt. So lange die Kristallstruktur konstant bleibt, kann dies nur durch eine Verringerung der Molekülsymmetrie infolge einer das Symmetrieverbot aufhebenden Fixierung der $\mathrm{CH}_{3}$-Gruppen erfolgen. Diese Fixierung ist auch für die geringe Linienbreite verantwortlich: Das Spektrum des Durol-Kristalls besteht auch bei $77^{\circ} \mathrm{K}$ noch aus sehr breiten Banden ${ }^{10}$.

Kurz vor Erreichen der Temperatur des flüssigen $\mathrm{O}_{2}$ ändert sich das Spektrum sprunghaft (Abb. 4 b) : Man beobachtet eine diskontinuierliche Verschiebung um $60 \mathrm{~cm}^{-1}$ nach Rot, eine Änderung in der Intensitätsverteilung auf die Schwingungsbanden (besonders eine Schwächung von 0.0) und eine entscheidende Änderung der Polarisations-Verhältnisse.

Diese Änderung wurde zuerst von Broude ${ }^{6}$ beobachtet und als Phasenänderung bei ca. $110^{\circ} \mathrm{K}$ gedeutet. Sie ist von einer Veränderung der Doppelbrechung (beobachtbar an der Farbänderung zwischen gekreuzten Polarisatoren) und von einem Zerspringen der Einkristalle längs der parallel $b$ verlaufenden Spaltrisse begleitet. Die Bruchstücke nehmen bei Temperatur-Erhöhung wieder die alte Struktur an.

In keinem Falle gelang es, Kristalle ohne Phasenumwandlung wesentlich zu unterkühlen, wenn ihre Dicke größer als etwa $4 \mu$ war. Auch bei unmittelbarem Eintauchen in flüssigen Stickstoff zerfielen

* Abb. 4 a-c auf Tafel S. 340 a.

10 H. C. Wolf, Z. Naturforschg. 11 a, 797 [1956]. die Kristalle in der für die Phasenumwandlung charakteristischen Weise.

Dagegen gelang diese Unterkühlung ohne jede Schwierigkeit bei dünneren Kristallen. Bei weniger als etwa $3 \mu$ Dicke beobacheten wir in keinem Falle die Phasenumwandlung. Vermutlich würde man diese jedoch auch hier erreichen, wenn man beim Abkühlen die Umwandlungstemperatur hinreichend langsam unterschritte. Offenbar stehen die Geschwindigkeiten der Umwandlung und der Abkühlung durch das Kristall-Volumen hindurch in Konkurrenz: bei dünneren Kristallen überwiegt letztere und man erreicht die Unterkühlung.

Es wurden also die Absorptionsspektren von 2 Phasen gemessen:

1. der Hochtemperaturphase (HT-Phase)

bei $77^{\circ} \mathrm{K}$ an Kristallen von 0.5 bis ca. $4 \mu$,

2. der Tieftemperaturphase (TT-Phase)

bei $77^{\circ} \mathrm{K}$ an Kristallen von 3 bis ca. $100 \mu$.

Unsere Zuordnung der beobachteten Spektren zu den beiden Phasen stimmt mit der von Broude ${ }^{6}$ überein. Die von McClure und $\mathrm{S}_{\text {chnepp }}{ }^{7}$ gemessenen Spektren entsprechen dagegen nach unseren Beobachtungen der TT-Phase ${ }^{* *}$.

\section{Absorptionsspektren}

Die beobachteten Absorptionsspektren sind in den Abb. 3, 4 und 5 und in Tab. 4 zusammengestellt. Wegen der großen Zahl der Linien wurde keine vollständige quantitative Absorptionskurve gezeichnet. In Abb. 3 sind nur die 0.0.-Linien und der Verlauf des molaren dekadischen Extinktionskoeffizienten (als Umhüllende der übrigen Absorptionslinien) dargestellt. Die Registrierphotometerkurven der Abb. 5 sind in Tab. 4 ausgewertet. Die Zuordnung der Molekülschwingungsfrequenzen erfolgte wie üblich durch Aufsuchen von Serien und Vergleich mit Raman-Frequenzen.

IV. Die Fluoreszenzspektren (Abb. 6) sind bei den beiden Phasen den Absorptionsspektren genau spiegelbildlich mit der 0.0-Linie als Mittelpunkt. Die beobachteten Banden bzw. Linien gibt Tab. 5 und 6 . Die HT-Fluoreszenz konnte mit hinreichender Intensität nur an dickeren Kristallen oberhalb des Umwandlungspunktes, d. h. bei ca. $100{ }^{\circ} \mathrm{K}$ beobachtet

\footnotetext{
** A n m. b. d. K o r r. : Zu dem gleichen Ergebnis kommt O. Schnepp in einer neuen Arbeit, die er dem Verfasser freundlicherweise vor der Veröffentlichung im Manuskript zugänglich machte.
} 


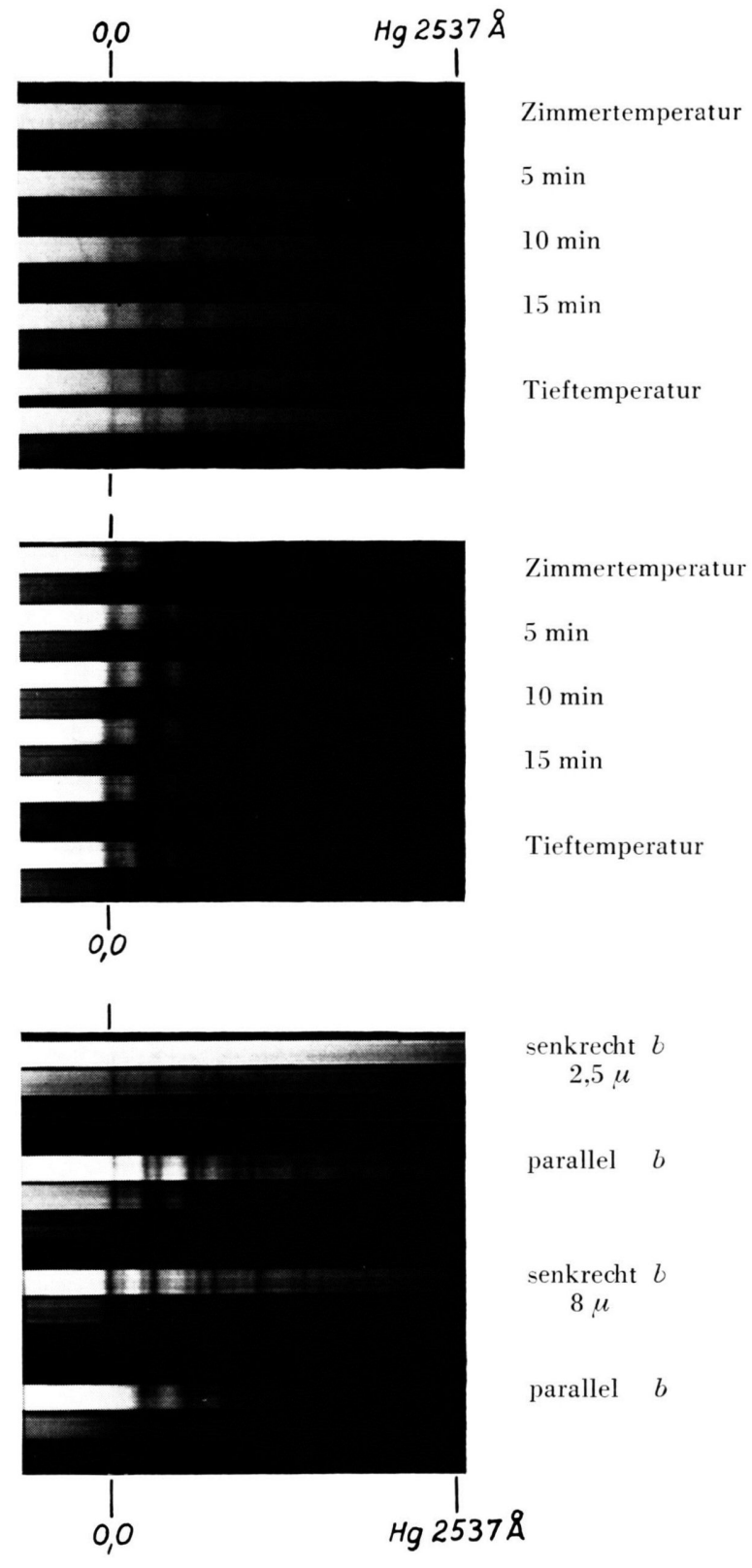

4. a) Dicke ca. $1,5 \mu$.

Aufnahmen während des Abkühlens alle 5 min. Keine Phasen. umwandlung.
4. b) Dicke ca. $5 \mu$.

Aufnahmen während des Abkühlens, alle $5 \mathrm{~min}$.

Phasenumwandlung nach 15 min.
4. c) Spektren der beiden Phasen bei Tieftemperatur. Elektr. Lichtvektor parallel und senkrecht zur Achse $b$.

Abb. 4. Absorptionsspektren von Hexamethylbenzol-Kristallen zur Erläuterung der Phasenumwandlung in dickeren Kristallen. 


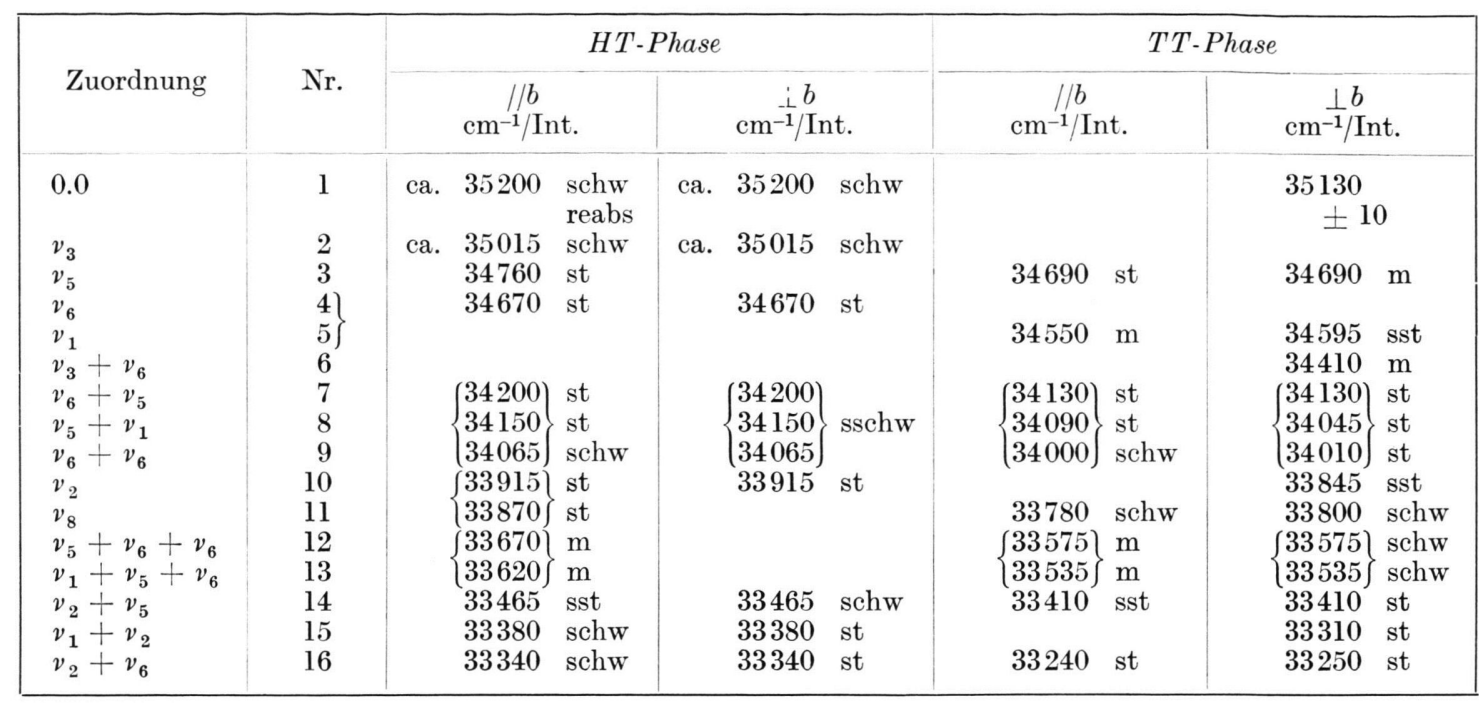

Tab. 5. Fluoreszenzspektrum von kristallisiertem Hexamethylbenzol. - Die Zahlenwerte für die Frequenzen $v_{1} \ldots v_{8}$ enthält Tab. 6. - Intensitäten: sst $=$ sehr stark, st $=$ stark, $\mathrm{m}=$ mittel, $\mathrm{sch} w=$ schwach.

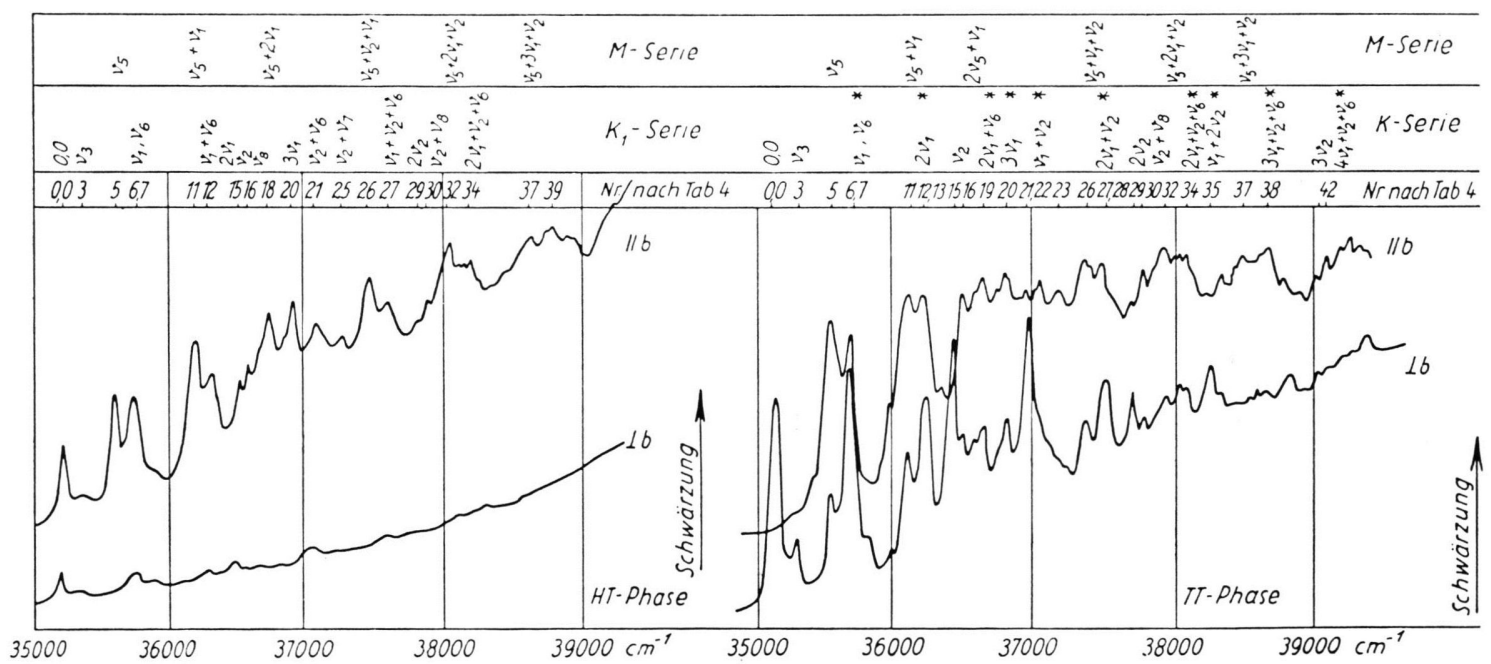

Abb. 5. Absorptionsspektrum von HMB-Kristallen in der $b c$-Ebene bei $77^{\circ} \mathrm{K}$, Registrierphotometerkurven, links $=\mathrm{HT}$-, rechts $=$ TT-Phase. - Oben: Einteilung der Linien in M- und K-Serie. Die mit einem Stern bezeichneten Linien gehören zur Serie $\mathrm{K}_{2}$.

werden. Die Banden sind deshalb weniger gut aufgelöst als die in den übrigen Spektren, die bei 77 oder $90^{\circ} \mathrm{K}$ gemessen wurden. Schematisch ergibt sich für die relative Intensität der aktiven Molekülschwingungen in den verschiedenen Spektren das in Abb. 7 dargestellte Bild.

V. Zur Ergänzung der hier mitgeteilten Spektren können die Absorptions- und Fluoreszenzspektren der TT-Phase bei $20{ }^{\circ} \mathrm{K}$ von Schnepp und McClure ${ }^{7}$ dienen. Die tiefere Temperatur ermöglicht eine bes- sere Auflösung der Spektren, im übrigen stimmen sie jedoch mit den eigenen innerhalb der Fehlergrenzen überein.

Broude $^{6}$ und Schnepp und McClure ${ }^{7}$ berichten ferner über Messungen an der $a b$-Ebene. Danach stimmt in dieser Ebene bei der TT-Phase die Absorption parallel $a$ und parallel $b$ völlig mit der $b$-Absorption in der $b c$-Ebene überein, lediglich die 0.0-Bande parallel $a$ scheint wesentlich schwächer zu sein als die parallel $b$. 


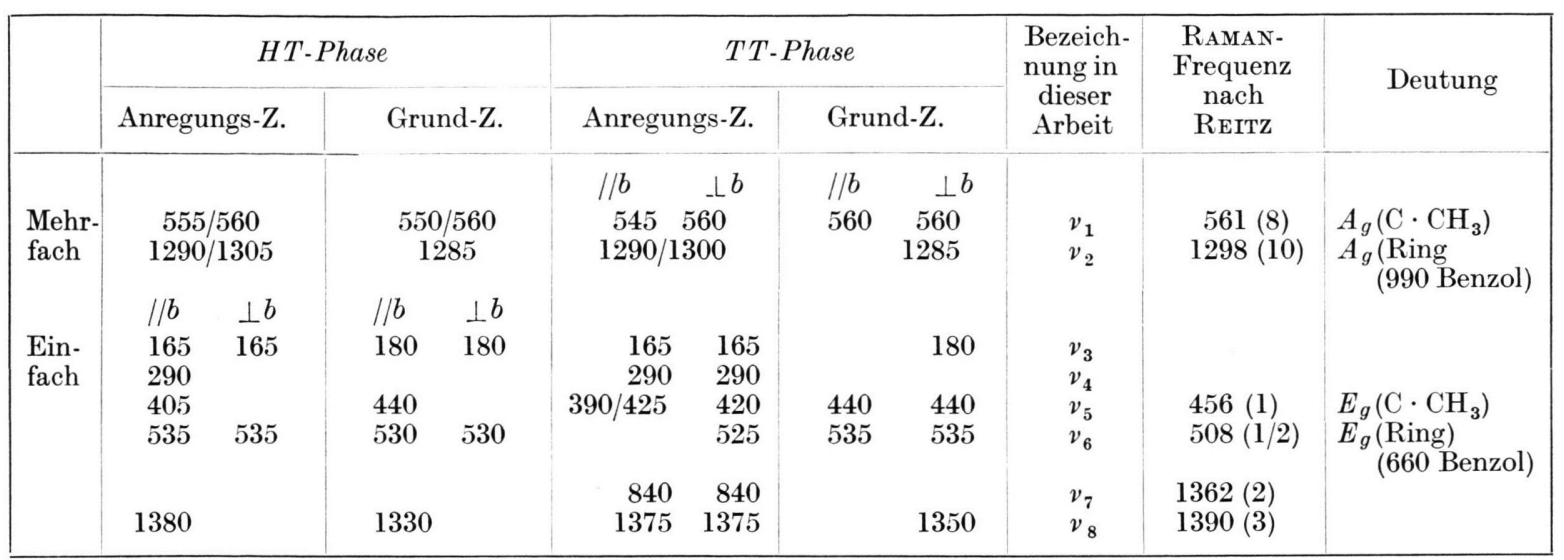

Tab. 6. Mittelwerte der Schwingungsfrequenzen des Hexamethylbenzol-Moleküls im Kristall und Vergleich mit Raman-Frequenzen.
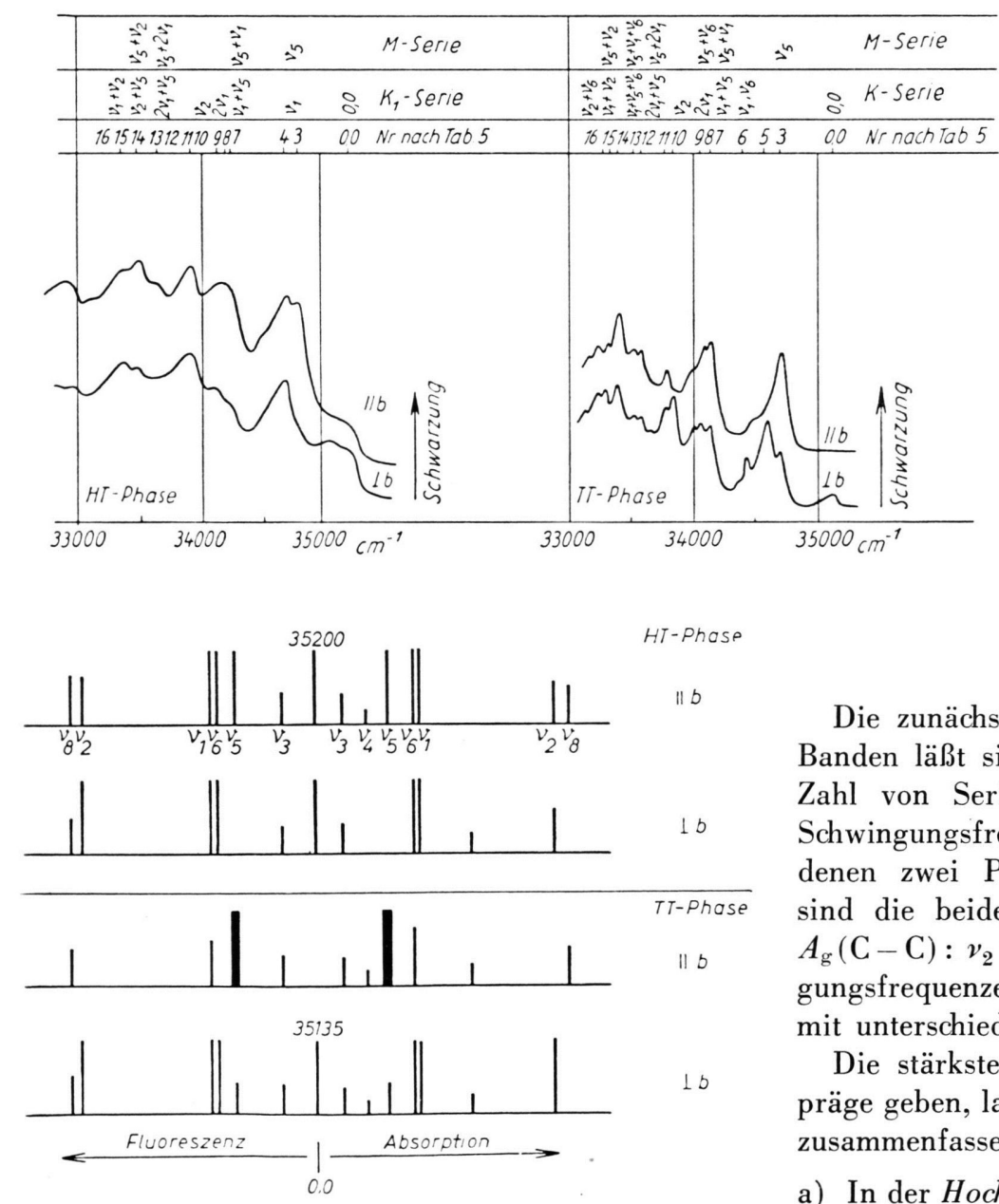

Abb. 7. Schwingungsstruktur in den HMB-Spektren. Eingetragen sind nur einfache Schwingungen, keine Kombinationen. zusammenfassen:
Abb. 6. Fluoreszenzspektrum von HMB-Kristallen in der $b c$-Ebene, Registrierphotometerkurven, links $=$ HT-Phase bei ca. $110^{\circ} \mathrm{K}$, rechts $=$ TT-Phase bei ca. $77^{\circ} \mathrm{K}$.

\section{Diskussion}

Die zunächst verwirrende Fülle von Linien und Banden läßt sich doch recht sicher in eine geringe Zahl von Serien einordnen. Dies gelingt mit 8 Schwingungsfrequenzen des Moleküls $v_{1}-v_{8}$, von denen zwei Perioden bilden $\left(v_{1}\right.$ und $\left.v_{2}\right)$. Dies sind die beiden totalsymmetrischen Schwingungen $A_{\mathrm{g}}(\mathrm{C}-\mathrm{C}): v_{2}$ und $A_{\mathrm{g}}\left(\mathrm{C}-\mathrm{CH}_{3}\right): v_{1}$. Die 8 Schwingungsfrequenzen sind in den verschiedenen Spektren mit unterschiedlicher Intensität angeregt (Abb. 7).

Die stärksten Linien, die den Spektren das Gepräge geben, lassen sich in folgender Weise in Serien

a) In der Hochtemperaturphase beobachtet man parallel b die M-Serie (s. S. 339) 
M-Serie $=0.0$ (verboten $) \pm v_{5} \pm n_{1} v_{1} \pm n_{2} v_{2} \pm m\left(v_{3} \ldots 8\right)$ und eine (nur im Kristall, nicht im Lösungsspektrum beobachtete, deshalb K-Serie genannte) $\mathrm{K}_{1}$-Serie.

$\mathrm{K}_{1}$-Serie $=0.0($ erlaubt $) \pm n_{1} v_{1} \pm n_{2} v_{2} \pm m\left(v_{3 \ldots 8}\right)$ mit $n_{1,2}=0,1,2,3, \ldots$ und $m=0$ oder 1 .

senkrecht $b$ nur die $\mathrm{K}_{1}$-Serie.

Für die Intensiätsverhältnisse gilt etwa:

$\frac{\text { M-Serie parallel } b}{\text { M-Serie senkrecht } b}=1000 ; \frac{\text { K-Serie parallel } b}{\text { K-Serie senkrecht } b}=10$.

\section{Deutung:}

Man kann die Absorption des „freien“ Moleküls von der durch das Kristallfeld induzierten trennen. Die Molekülabsorption und -fluoreszenz (M-Serie) liegt nur in der Molekülebene. Sie entspricht einem Symmetrie-verbotenen schwachen Übergang, der durch eine $E_{\mathrm{g}}$-Schwingung $\left(v_{5}\right)$ seine Intensität erhält. Die Kristallabsorption und -fluoreszenz (KSerie) ist erlaubt; sie hat eine stärkere Komponente in der Molekülebene und eine schwächere Komponente senkrecht dazu. Da die Symmetrie des HMBKristalls alle für das HMB-Molekül geltenden Übergangsverbote aufhebt, kann die Kristallsymmetrie für die Struktur der $K_{1}$-Serie verantwortlich sein. Möglich ist jedoch auch eine Verringerung der HMBMolekülsymmetrie im Kristall durch Fixierung der $\mathrm{CH}_{3}$-Gruppen.

Die 0.0-Linie spaltet nicht auf. Damit wird die Erwartung der Theorie der Molekülkristallspektren ${ }^{10}$ bestätigt, wonach im Kristall mit nur einem Molekül in der Einheitszelle keine Davydov-Aufspaltung erfolgt.

b) In der Tieftemperaturphase beobachtet man

parallel $b$ stark die M-Serie

etwas schwächer eine K-Serie (Teilserie von $\mathrm{K}_{1}$ ) : $\mathrm{K}_{2}$-Serie $=0.0($ verboten $) \pm n v_{1}$

senkrecht $b$ im wesentlichen nur die $\mathrm{K}_{1}$-Serie.

Dabei ist die $\mathrm{K}_{1}$-Serie so stark, daß das Intensitätsverhältnis der Gesamtabsorption, bzw. -fluoreszenz sich parallel $b$ : senkrecht $b$ wie $3: 1$ verhält.

\section{Deutung:}

Die Molekülabsorption (M-Serie) bleibt mehr oder weniger ungeändert, dagegen ändert sich die Kristallabsorption. Die $\mathrm{K}_{1}$-Serie ist jetzt fast vollständig senkrecht $b$ polarisiert.
Über die neue Kristallstruktur ist wenig bekannt. Man stellt fest, daß die Kristalle bei der Phasenumwandlung zwar längs präformierter Spaltrisse parallel $b$ springen, im wesentlichen aber erhalten bleiben, ferner daß die Auslöschrichtungen zwischen gekreuzten Nicols und die Lagen der optischen Achsen im Kristall wenig oder gar nicht verändert werden. Die Absorptions- und Fluoreszenzspektren geben weitere Aussagen:

1. Die Zahl der Moleküle in der Einheitszelle bleibt wahrscheinlich erhalten $(Z=1)$, da eine DAvYDov-Aufspaltung weiterhin fehlt.

2. Die Orientierung der Molekülebene bleibt fast unverändert, da die M-Serie auch weiterhin parallel $b$ polarisiert ist.

Diese Beobachtungen legen die Vermutung nahe, daß die Phasenänderung hauptsächlich durch ein Umklappen der - im Kristall fixierten - $\mathrm{CH}_{3}$ Gruppen in eine neue Lage gekennzeichnet ist, während die Lage der Ringe erhalten bleibt. Durch dieses Umklappen ändert sich die Molekülsymmetrie.

Unter diesen Voraussetzungen sind die Überlegungen von Schnepp und McClure ${ }^{7}$ über die Symmetrie des HMB-Moleküls im Kristall anwendbar; sie ergeben für die TT-Phase die Molekülsymmetrie $\mathrm{S}_{6}$. Über die Molekülsymmetrie und damit über die Lage der $\mathrm{CH}_{3}$-Gruppen in der HT-Phase sind keine Aussagen möglich.

Die allgemeine Verschiebung des TT-Spektrums um $60 \mathrm{~cm}^{-1}$ nach Rot gegenüber der HT-Phase ist einfach eine Folge der dichteren Packung.

Nach dieser Erklärung der Haupteigenschaften der Spektren lassen sich auch weitere Einzelheiten verstehen: die $\mathrm{K}_{2}$-Serie und die Inkonstanz der Schwingungsfrequenzen in den verschiedenen Spektren.

Wenn die Molekülsymmetrie durch die $\mathrm{CH}_{3}$-Gruppen verkleinert wird, kann von den beiden totalsymmetrischen $A_{\mathrm{g}}$-Schwingungen die C.C-Schwingung $\left(v_{2}\right)$ totalsymmetrisch bleiben, während das für die $\mathrm{C} \cdot \mathrm{CH}_{3}$-Schwingung nicht mehr gilt. Das hat zur Folge, daß auch bei verbotenem 0.0-Übergang die Schwingung $v_{1}$ erlaubt und als Ursprung der Serie $K_{2}$ im TT-Spektrum parallel $b$ wirksam sein kann. Die $K_{2}$-Serie ist dann eine Teilserie von $K_{1}$.

Eine andere Möglichkeit zur Erklärung $\operatorname{der} \mathrm{K}_{2}$ Serie kann nicht ausgeschlossen werden; man kann als Formel für die $\mathrm{K}_{2}$-Serie auch ansetzen: 0.0 (verboten) $\pm v_{6} \pm n v_{1} ; v_{6}$ wäre dabei die $E_{\mathrm{g}}\left(\mathrm{C} \cdot \mathrm{CH}_{3}\right)$. Schwingung. die dem Symmetrie-verbotenen Über- 
gang seine Intensität gibt. Die Serie wäre dann also eine Molekülserie und als $\mathrm{M}_{2}$-Serie zu bezeichnen.

Damit ist die Schwingungsstruktur in den wesentlichen Zügen erklärt. Unsicherheiten bestehen noch bei der genauen Festlegung der Schwingungsfrequenzen. Diese Unsicherheiten haben verschiedene Gründe:

1. muß man offenbar damit rechnen, daß Unterschiede in der sterischen Anordnung der $\mathrm{CH}_{3}$-Gruppen auch Unterschiede in den Schwingungsfrequenzen ergeben. So ist es zu erklären, warum die gleichen Schwingungen in verschiedenen Spektren etwas verschiedene Frequenzen aufweisen können,

2. sind auch bei $20^{\circ} \mathrm{K}$ die Gitterschwingungen noch nicht eindeutig analysierbar.

Die Mittelwerte der Schwingungsfrequenzen, wie sie sich aus der Analyse in Tab. 4 und 5 ergeben,

11 A. W. Reitz, Z. phys. Chem. B 46, 181 [1940]. - J. W. Murray u. D. H. Andrews, J. Chem. Phys. 2, 120 [1934]. sind in Tab. 6 zusammengestellt und mit den bekannten Raman-Daten ${ }^{11}$ verglichen.

Die wichtigsten Unsicherheiten sind folgende:

1. Es ist nicht sicher, ob der unterschiedliche Wert für $v_{1}$ parallel und senkrecht $b$ in der TT-Phase $\left(545-560 \mathrm{~cm}^{-1}\right)$ reell oder durch Gitterschwingungen vorgetäuscht ist. Weiter ist nicht sicher, ob es sich bei den mit $v_{1}$ und $v_{6}$ bezeichneten Frequenzen wirklich um zwei verschiedene Schwingungen handelt, oder um dieselbe Schwingung für zwei verschiedene Anordnungen der $\mathrm{CH}_{3}$-Gruppen. Deshalb kann zwischen den beiden Erklärungsmöglichkeiten für die $\mathrm{K}_{2}$-Serie noch nicht entschieden werden.

2. Für die Deformationsschwingung $v_{5}$, den Ursprung der M-Serie, kann man in der TT-Phase keinen sicheren Frequenzwert angeben, da für sie die Bandenbreite zu groß ist.

Diese Unsicherheiten können durch weitere Analyse der Spektren bei tiefsten Temperaturen mit höchster Auflösung aufgeklärt werden.

\section{NOTIZEN}

\section{Klassische Lösungen einer Heisenbergschen nichtlinearen Feldgleichung}

\author{
Von K. Just
}

Institut für Theoretische Physik der Freien Universität Berlin (Z. Naturforschg. 13 a, 345-346 [1958]; eingeg. am 8. März 1958)

In einer älteren Arbeit zu seiner nichtlinearen Feldtheorie erhielt Heisenberg ${ }^{1}$ für einen Erwartungswert $c$ die Gleichung ${ }^{2}$

$$
\gamma^{v} c_{\mid v}=(\bar{c} c) c+\varkappa c \quad \text { mit } \quad \varkappa=\text { const }>0 .
$$

Fragt man nach deren „klassischen“ Lösungen von der speziellen Form

$$
c(x)=s^{-3 / 4}\left(\sqrt{s} F+\gamma^{v} x_{v} G\right) a, \quad s=-x_{r} x^{v}>0,
$$

worin $a$ ein konstanter Spinor, $F$ und $G$ reelle Funktionen von $s$ seien, so ergibt sich

$$
\left.\begin{array}{l}
{ }_{2}^{1} F-v F^{\prime}=v G+A\left(F^{2}+G^{2}\right) G, \\
{ }_{2}^{5} G+v G^{\prime}=v F+A\left(F^{2}+G^{2}\right) F
\end{array}\right\}
$$

mit

$$
v=\varkappa \sqrt{s}, \quad F^{\prime}=\mathrm{d} F / \mathrm{d} v, \quad A=a a=\text { const. }
$$

1 W. Heisenberg, Z. Naturforschg. 9 a, 292 [1954].

2 Die Änderung der Vorzeichen gegenüber Formel (28) aus ${ }^{1}$ rührt von Heisenberg ${ }^{3}$ her; seine Konstante $l$ ist hier als Längen-Einheit benutzt.
Versuchen wir den Ansatz

$$
F=r(v) \cos \varphi(v), \quad G=r(v) \sin \varphi(v),
$$

so liefert (3) nach einiger Rechnung:

und

$$
\left.\begin{array}{l}
v \varphi^{\prime}=v-{ }_{2}^{3} \sin 2 \varphi+A r^{2} \\
v r^{\prime}=\left(\begin{array}{l}
3 \\
2
\end{array} \operatorname{ccs} 2 \varphi-1\right) r .
\end{array}\right\}
$$

Statt dieser strengen Gleichungen betrachten wir jedoch nur die Näherungen für sehr große Werte von $v$ oder $r$. Im ersten Falle wird $\varphi^{\prime}=1, r=$ const $\cdot v^{-1}$,

$$
\left.\begin{array}{lrl}
\text { also } & r=\mu s^{-1 / 2}, & \varphi=\varkappa \sqrt{s}-\lambda \\
\text { für } & \varkappa V s \gg 1,|A| r^{2} & \text { mit } \quad \mu, \lambda=\text { const },
\end{array}\right\}
$$

im zweiten erhalten wir schließlich:

$$
\left.\begin{array}{c}
r=\alpha s^{-1 / 2}, \quad \varphi=\beta-{ }_{2}^{1} A \alpha^{2} s^{-1} \\
A \mid r^{2} \gg 1, \chi \sqrt{s} \quad \text { mit } \quad \alpha, \beta=\text { const . }
\end{array}\right\}
$$

für

In der $(F, G)$-Ebene stellt (5) mit der Näherung (7) eine Spirale dar, die sich im positiven Sinne um den Ursprung windet und für $s \rightarrow \infty$ darin einmündet. Mit der Näherung (8) ergibt sich ebenfalls eine Spirale, die für $s \rightarrow 0$ unbegrenzt wächst und deren Dreh. sinn im Falle $A>0$ derselbe ist wie mit (7), bei

3 W. Heisenberg, Rev. Mod. Phys. 29, 269 [1957], Fußnote. 